\title{
Determination of free fatty acids in palm oil by near-infrared reflectance spectroscopy
}

\begin{abstract}
A near-infrared (NIR) spectroscopy calibration was developed for the determination of free fatty acids (FFA) in crude palm oil and its fractions based on the NIR reflectance approach. A range of FFA concentrations was prepared by hydrolyzing oil with $0.15 \%$ (w/w) lipase in an incubator at $60^{\circ} \mathrm{C}(200 \mathrm{rpm})$. Sample preparation was performed in Dutch cup, and the spectra were measured in duplicate for each sample. The optimized calibration models were constructed with multiple linear regression analysis based on $\mathrm{C}=\mathrm{O}$ overtone regions from 1850 ï $2050 \mathrm{~nm}$. The best wavelength combinations were 1882, 2010, and $2040 \mathrm{~nm}$. Multiple correlation coefficients squared (R2) were: 0.994 for crude palm oil, 0.961 for refinedbleached-deodorized (RBD) palm olein, and 0.971 for RBD palm oil. Calibrations were validated with an independent set of 81010 samples. R2 of validation were $0.997,0.943$, and 0.945 , respectively. The developed method was rapid, with a total analysis time of $5 \mathrm{~min}$, and environmentally friendly, and its accuracy was generally good for raw-material quality control.
\end{abstract}

Keyword: Free fatty acids; MLR; NIR; Palm oil; Spectroscopy 